\title{
Clusterização do perfil de adolescentes escolares com predisposição ao uso de substância psicoativas
}

\author{
Clustering the profile of school adolescents predisposed to psychoactive substance use
}

Agrupación del perfil de adolescents escolares predispuestos al consume de substancias psicoativas

Recebido: 31/01/2021 | Revisado: 05/02/2021 | Aceito: 11/02/2021 | Publicado: 19/02/2021

Dayane Diniz Martins

ORCID: https://orcid.org/0000-0002-6903-364X Universidade do Estado do Pará, Brasil E-mail: dayane.martins@aluno.uepa.br

Laís Balla Lucena

ORCID: https://orcid.org/0000-0002-7068-9988 Universidade do Estado do Pará, Brasil

E-mail: lais.lucena@aluno.uepa.br

Athos Ricardo Moraes Bastos Damasceno

ORCID: https://orcid.org/0000-0001-7012-4728

Universidade do Estado do Pará, Brasil

E-mail: athos.damasceno@aluno.uepa.br

Hugo Siqueira

ORCID: https://orcid.org/0000-0002-1278-4602

Universidade Tecnológica Federal do Paraná, Brasil

E-mail: hugosiqueira@utfpr.edu.br

Yara de Souza Tadano

ORCID: https://orcid.org/0000-0002-3975-3419

Universidade Tecnológica Federal do Paraná, Brasil

E-mail:yaratadano@utfpr@edu.br

Ivete Furtado Ribeiro Caldas

ORCID: https://orcid.org/0000-0002-2095-101X Universidade do Estado do Pará, Brasil

E-mail: ivetecaldas@uepa.br

\begin{abstract}
Resumo
A adolescência é um período de transformações neurobiológicas e comportamentais. O desenvolvimento moral (DM), ainda em amadurecimento nesta fase, é importante na tomada de decisão, como o uso ou não de SPA. Objetiva-se aplicar algoritmos de clusterização para agrupamento de perfil de adolescentes escolares com tendência ao uso de SPAs, baseado nos fatores socioenonômicos e no nível de DM. Trata-se de um estudo transversal com amostra por conveniência. Participaram do estudo 100 adolescentes, entre 12 a 18 anos, de ambos os sexos e regularmente matriculados no ensino médio. Foram utilizados o questionário socioeconômico, CRAFFT/CESARE (acrônimo de Car; Relax; Alone; Forget; Family/Friends; Trouble) e o teste de competência moral. Foi utilizado o método de clusterização para análise de dados. Quanto aos resultados encontrados, foi verificado que onze (11.0\%) apresentam risco para o uso, abuso ou dependência de SPAs, $6.0 \%$, suspeita de dependência de SPAs e $2.0 \%$, risco de dependência de SPAs. No dilema do médico, houve predomínio dos estágio 1 e 2, do juiz, 5 e 6 e operário, 4 e 5 . O método Fuzzy C-Means mostrou-se mais adequado, com alta correlação com as respostas fornecidas pelo teste CRAFFT/CESARE. Portanto, os métodos de clusterização apresentam resultados com valores próximos aos obtidos pelo teste CRAFFT/ CESARE quanto ao perfil de adolescentes escolares com predisposição ao uso de SPAs, contribuindo na relação dos fatores determinantes na tomada de decisão nessa etapa da vida.
\end{abstract}

Palavras-chave: Adolescente; Desenvolvimento moral; Questionário; Abuso de substâncias psicoativas.

\begin{abstract}
Adolescence is a period of neurobiological and behavioral changes. Moral development (DM), still maturing at this stage, is important in decision making, such as the use or not of SPA. The objective is to apply clustering algorithms to group the profile of school adolescents with a tendency to use SPAs, based on socio-economic factors and the level of DM. This is a cross-sectional study with a convenience sample. 100 adolescents, between 12 and 18 years old, of both sexes and regularly enrolled in high school participated in the study. The socioeconomic questionnaire, CRAFFT / CESARE (acronym for Car; Relax; Alone; Forget; Family / Friends; Trouble) and the moral competence test were
\end{abstract}


used. The clustering method was used for data analysis. As for the results found, it was possible to confirm that eleven $(11.0 \%)$ are at risk for the use, abuse or dependence of SPAs, $6.0 \%$, suspected of dependence on SPAs and $2.0 \%$, risk of dependence on SPAs. In the physician's dilemma, there was a predominance of stages 1 and 2, of the judge, 5 and 6 and worker, 4 and 5. The Fuzzy C-Means method was more appropriate, with a high correlation with the answers provided by the CRAFFT / CESARE test. Therefore, the clustering methods present results with values close to those obtained by the CRAFFT / CESARE test regarding the profile of school adolescents predisposed to the use of SPAs, contributing to the list of determining factors in decision making at this stage of life.

Keywords: Adolescent; Moral development; Quiz; Abuse of psychoactive substances.

\section{Resumen}

La adolescencia es un período de cambios neurobiológicos y de comportamiento. El desarrollo moral (DM), que aún está madurando en esta etapa, es importante en la toma de decisiones, como el uso o no de SPA. El objetivo es aplicar algoritmos de clustering para agrupar el perfil de adolescentes escolares con tendencia a utilizar SPA, en función de factores socioeconómicos y el nivel de DM. Se trata de un estudio transversal con una muestra de conveniencia. El estudio incluyó a 100 adolescentes, entre 12 y 18 años, de ambos sexos y matriculados regularmente en la escuela secundaria. Se utilizó el cuestionario socioeconómico CRAFFT / CESARE (siglas de Car; Relax; Alone; Forget; Family / Friends; Trouble) y la prueba de competencia moral. Se utilizó el método de agrupamiento para el análisis de datos. En cuanto a los resultados encontrados, se pudo confirmar que once $(11,0 \%)$ tienen riesgo de uso, abuso o dependencia de SPA, 6,0\% sospecha de dependencia de SPA y 2,0\% riesgo de dependencia de SPA. En el dilema del médico, predominaron las etapas 1 y 2, del juez, 5 y 6 y trabajador, 4 y 5 . El método Fuzzy C-Means resultó ser más apropiado, con una alta correlación con las respuestas proporcionadas por la prueba CRAFFT / CESARE. Por lo tanto, los métodos de agrupamiento presentan resultados con valores cercanos a los obtenidos por la prueba CRAFFT / CESARE en cuanto al perfil de adolescentes escolares predispuestos al uso de SPA, contribuyendo a la lista de factores determinantes en la toma de decisiones en esta etapa de la vida.

Palabras clave: Adolescente; Desarrollo moral; Examen; Abuso de sustancias psicoactivas.

\section{Introdução}

O desenvolvimento humano se inicia na concepção e continua por toda a vida. O padrão é complexo por ser produto dos processos biológico, cognitivo e socioemocional, e pode ser influenciado por fatores biopsíquicos e socioculturais nos quais os adolescentes estão inseridos (Marriel, Assis, Avanci, \& Oliveira, 2013). Adolescência, do latim adolescere que significa crescer, desenvolver-se, tornar-se jovem, tem sido definida como um período biológico, psicológico e social compreendido entre os 10 e os 18 anos (Schoen-Ferreira, Aznar-farias, \& Silvares, 2010). No mundo, existe aproximadamente 1 bilhão de pessoas nessa faixa etária, representando cerca de $20 \%$ da população de todo o planeta. A adolescência é um período de transformações neurobiológicas e comportamentais, essenciais para formação do indivíduo. Nessa fase da vida, há maior autonomia nas circulações sociais, na auto-percepção, auto-imagem e aceitação social, gerando maior necessidade de novas experiências e estímulos (Pagliace, Maftum, Lacerda, Kantorski, Nimtz, \& Brusamarello, 2019; Pereira \& Azevedo, 2019; Freitas \& Souza, 2020).

Embora a maioria das pesquisas de desenvolvimento sobre o funcionamento cognitivo e psicossocial durante a adolescência envolva estudos psicológicos, estudos em neurociência esclarecem as bases neurais sobre essa fase da vida, incluindo os comportamentos típicos do adolescente, como comportamento de risco e impulsividade (Colver \& Longwell, 2013; Cohen \& Casey, 2014). Tais comportamentos são produtos da interação entre as mudanças de dois sistemas neurobiológicos distintos que estão em pleno amadurecimento nesse período da vida: o sistema socioemocional ou moral, localizado em áreas límbicas e paralímbicas do cérebro, e o sistema de controle cognitivo, que é composto principalmente pelos córtices parietal lateral e pré-frontal, responsável pela tomada de decisões e controle do impulso (Andrade et al., 2017; Ramos, 2019). Nesse período os circuitos neurais ficam mais eficientes e específicos, além de ocorrer um aumento significativo da dopamina, estimulando a busca de sensações em virtude do amadurecimento da via de recompensa (Ramos, 2019; Granito \& Zaher-Rutherford, 2019).

A via de recompensa está associada a comportamentos que geram sensação de prazer (p.ex. comer, beber, fazer sexo) e funciona como reforço positivo para que o comportamento seja repetido. Esses comportamentos são essenciais para a 
sobrevivência da nossa espécie. Entretanto, outros estímulos também ativam a via de recompensa (p.ex. a admiração dos nossos pares, um comportamento arriscado bem-sucedido e o uso de substâncias psicoativas - SPAs) mesmo quando associados com o risco de consequências negativas (Brasil,2017). As SPAs (maconha, cocaína ou inalantes) têm a capacidade de produzir alterações nas sensações, no grau de consciência e no estado emocional de quem as consome, tendo como base modificações ao nível do Sistema Nervoso Central (SNC) (Coutinho, et al., 2016; Schlindwein-Zanini \& Sotili, 2019).

Diante desse panorama, no período da adolescência, o desenvolvimento moral ainda está em fase de amadurecimento e consiste em um processo racional que acompanha a maturação cognitiva, possuindo diferentes níveis, nos quais o indivíduo progride à medida que abandona o pensamento egocêntrico (Kohlberg, 1981; Bataglia, Morais, \& Lepre, 2010; Nunes \& Bataglia, 2019). As habilidades que envolve o desenvolvimento moral são desenvolvidas ao longo da vida do indivíduo e são imprescindíveis para tomada de decisão e/ou julgamento em situações, como o uso ou não de SPAs (Granito \& ZaherRutherford, 2019).

Dessa forma, a compreensão entre o desenvolvimento moral, a tomada de decisão e o uso de SPAs na adolescência é de fundamental importância, pois em conjunto com as alterações físicas, o desenvolvimento cognitivo e emocional desses jovens também sofre drásticas modificações nessa fase (Carbonário, 2018; Velho, Quintana, \& Rossi, 2014). Dentro dessa perspectiva, este estudo tem como objetivo a aplicação de algoritmos de clusterização para agrupamento de perfil de adolescentes escolares com predisposição ao uso de SPAs, baseado nos fatores socioenonômicos e no nível desenvolvimento moral.

\section{Metodologia}

Trata-se de um estudo quantitativo, transversal com amostra por conveniência (Pereira, 2018). Os dados foram coletados na Escola Estadual de Ensino Médio Prof. Anízio Teixeira, no município de Marabá, Pará, localizado no sudeste paraense, distando cerca de 485 quilômetros da capital Belém e ocupando uma área de 15.128,058 quilômetros. O período de coleta de dados ocorreu nos meses de agosto a outubro de 2019.

Participaram do estudo 100 adolescentes regularmente matriculados no ensino médio. Foram incluídos alunos entre 12 a 18 anos, de ambos os sexos, que aceitaram participar da pesquisa assinando o Termo de Assentimento Livre e Esclarecido (TALE) e cujos responsáveis legais assinaram o Termo de Consentimento Livre e Esclarecido (TCLE). Foram excluídos alunos matriculados em outras escolas do município e que não estiveram presentes no dia da coleta de dados.

\subsection{Instrumentos}

Questionário socioeconômico: composto por 11 perguntas objetivas que acessavam informações sobre variáveis socioeconômicas dos estudantes (idade, sexo, se pratica exercícios físicos e religião, exerce de função remunerada e se pais e/ou responsáveis consomem bebidas alcoólicas), qualidade do relacionamento do estudante com seu responsável (pai e/ou mãe), e nível econômico familiar, baseado na Pesquisa de Orçamento Familiar (POF) do Instituto Brasileiro de Geografia e Estatística (IBGE) (Campos, Almeida, Garcia, \& Faria, 2011). Esse instrumento objetiva avaliar a situação social e econômica dos estudantes e de sua família. Questionário CRAFFT/ CESARE (acrônimo de Car; Relax; Alone; Forget; Family/Friends; Trouble): utilizado na forma adaptada para o Brasil e internacionalmente recomendado para rastreamento de uso de substâncias psicoativas (SPAs) em adolescentes. Consiste num instrumento de triagem e não deve ser usado com critério diagnóstico (Pereira, et al., 2016). É composto por três perguntas de filtro sobre uso de bebidas alcoólicas, maconha ou outra droga (Parte A) e seis perguntas adicionais acerca de contexto e consequências relacionadas ao uso de SPAs (Parte B), cada resposta equivale a um ponto. Quanto a classificação de risco para o uso de SPAs é considerado CRAFFT/CESARE negativo (pontuação zero ou igual a um); positivo (maior ou igual a dois pontos); risco ao uso, abuso e dependência de SPA's (igual a 
três pontos); suspeita de dependência de SPAs (maior ou igual a quatro) e risco de dependência de SPAs (igual a seis pontos).

Teste de Competência Moral (Moral Competence Teste - MCT): instrumento adaptado para versão brasileira (Bataglia, 2010) que avalia a capacidade do sujeito em aplicar a estrutura de juízo moral em situações adversas, ou seja, o nível de competência moral dos participantes da pesquisa através do índice C (Lind, 2015). Três dilemas foram utilizados: Dilema do médico, juiz e operário, estes já validados nacional e internacionalmente (Bataglia et al., 2010). A estrutura dos três dilemas apresenta inicialmente uma pequena história sobre o assunto em questão, e o protagonista assume uma decisão para solucionar tal conflito em um respectivo contexto. Logo após a leitura de cada dilema, o estudante era instruído a responder sua opinião sobre a decisão do protagonista. Suas respostas seguem um formato Likert de $-3 \mathrm{a}+3$, variando de forte discordância até forte concordância. Em seguida, no mesmo sentido, 12 argumentos, sendo seis favoráveis e seis contrários a ação do protagonista, eram também respondidos, no formato likert de -4 a +4 , variando de rejeito completamente até aceito completamente o argumento.

\subsection{Procedimento}

\subsubsection{Coleta de dados}

Inicialmente foi solicitado à direção da escola que os pesquisadores participassem da reunião escolar de professores e responsáveis legais dos alunos, que ocorre bimestralmente de acordo com o calendário escolar, a fim de esclarecer os objetivos da pesquisa e a importância da participação dos alunos. Mediante assinatura no TCLE, os estudantes eram contatados e convidados a participarem da pesquisa respeitando as atividades escolares e o turno que estivessem matriculados.

Mediante a assinatura do TALE, iniciava-se a coleta de dados que foi conduzida em forma de entrevista individualizada. O pesquisador lia em voz compassada e em bom tom cada instrumento, e as perguntas relacionadas, o participante respondia oralmente. O pesquisador registrava assinalando com um " $\mathrm{X}$ " sobre a resposta indicada.

Uma sala foi disponibilizada nas dependências da escola para melhor comodidade e privacidade durante as entrevistas. A sala era arejada, com boa luminosidade, medindo 3x5m, aproximadamente, e continham 5 cadeiras e 1 mesa.

Primeiramente, foi coletado informações do CRAFFT/CESARE, seguido pelo teste de competência moral, e por fim, o questionário socioeconômico. Após a entrevista cada estudante foi identificado com um número (em substituição ao nome do aluno) que foram armazenados em um envelope. O tempo de cada entrevista foi de 30 minutos.

\subsubsection{Análise de dados}

Inicialmente foram computadas a frequência absoluta e relativa do perfil socioeconômico e a avaliação do CRAFFT/CESARE dos estudantes. Em seguida, foi calculado a preferência por estágio do desenvolvimento moral e o Índice C (nível da competência moral) para cada sujeito, e em seguida, o índice $\mathrm{C}$ médio para cada dilema. Os valores das variações de significância entre as médias dos Índice C total são 5 (alta significância) e 10 (muito alta significância). E a escala de referência corresponde de 0-9 pontos como competência moral muito baixa; 10-29 pontos para competência média (intervalo no qual se inserem a maioria dos indivíduos), e acima de 30 pontos para alta competência moral (Lind, 2000).

Por fim, foram aplicadas ferramentas de clusterização de dados que tem o objetivo segregar grupos com traços semelhantes e atribuí-los a clusters, ou seja, grupos de informações semelhantes. Os algoritmos de clusterização utilizados foram K-means, K-medoides e Fuzzy C-Means (FCM) (Figueiredo, et al., 2019). Para isso, uma base de dados original foi desenvolvida com a amostra do estudo $(\mathrm{n}=100)$. Os atributos (variáveis/dimensões) utilizados do questionário socioeconômicos foram: idade, sexo, prática de exercícios físicos, atividade remunerada, prática de alguma religião, consumo de bebida alcoólica pelos pais, relacionamento com o pai, com a mãe e classificação da classe econômica, totalizando 9 atributos. Seguidos pela preferência de estágios do desenvolvimento moral, nível de competência moral e a nota do 


\section{CRAFFT/CESARE.}

No processo de clusterização, foram realizadas simulações computacionais considerando parte ou a totalidade das informações disponíveis: 9 atributos socioeconômicos, 72 respostas aos dilemas, 3 resultados de preferência de estágio relativos a cada dilema e 3 valores do nível de competência moral, um para cada dilema. Assim, 3 cenários foram propostos com o intuito de verificar se todas as colunas realmente estavam contribuindo para a separação das classes. Sendo eles (1) atributos socioeconômicos e respostas aos dilemas (total de 81 atributos); (2) atributos socioeconômicos, respostas aos dilemas e preferência de estágio (total de 84 atributos) e (3) atributos socioeconômicos, respostas aos dilemas, preferência de estágio e nível de competência moral (total de 87 atributos).

Os métodos de clustering abordados são do tipo particional (K-Means e K-Medoides) e com sobreposição (FCM). No primeiro caso, um dado artificial nomeado como centroide é criado. Cada dado irá pertencer ao grupo em que o centroide está mais próximo. O algoritmo irá iterativamente posicioná-los de forma que a distância entre as amostras e os centroides seja mínima. O que muda entre K-Means e o K-Medoides e a forma de inicialização. Já o FCM cria uma matriz de pertinência, que mensura quanto um dado pode pertencer a cada classe.

Neste estudo foram utilizadas como métricas de avaliação para clusterização medidas que levam em consideração a distância interna, externa e outras definidas com base nestas: SSW (sum of squares within clusters), SSB (sum of squares between clusters) e Silhouette. O SSB calcula a distância entre os centroides e deve ser o maior possível. Dessa forma, os resultados mais expressivos em termos de clusterização são aqueles com menor valor para SSW, maior SSB e menor Silhouette. Ao final, comparamos os resultados alcançados pelos métodos de clustering e os resultados baseados no CRAFFT/CESARE.

A presente pesquisa foi aprovada pelo Comitê de Ética e Pesquisa com seres humanos da Universidade do Estado do Pará (UEPA) (Parecer n 3.278.940) respeitada a Resolução do Ministério da Saúde CNS/MS nº 466/12, com a participação condicionada à assinatura do Termo de Consentimento Livre e Esclarecido (TCLE) pelos pais e/ou responsáveis e do Termo de Assentimento Livre e Esclarecido (TALE) pelos estudantes.

\section{Resultados}

A Tabela 1 detalha as características socioeconômicas dos estudantes do estudo. Quanto a idade 72 (72.0\%) tem entre 16 e 18 anos de idade, 51 (51.0\%) são do sexo feminino, 23 (23.0\%) exercem função remunerada, 73 (73.0\%) praticam atividade física e $81(81.0 \%)$ alguma religião. A maioria dos pais ou responsáveis dos adolescentes 51 (51.0\%) consume bebidas alcoólicas e 90 (90.0\%) relatam ter bom relacionamento bom com a mãe, entretanto, com o pai, apenas 66 (66.0\%) afirmam ter bom relacionamento. Quanto a classificação socioeconômica, as classes B2 e C1 prevalecem com 30 (30.0\%) e 20 (20.0\%), respectivamente. 
Tabela 1 - Características socioeconômicas dos adolescentes do estudo.

$$
\mathbf{N}=\mathbf{1 0 0}
$$

Variáveis

\section{n}

$12-15$

Sexo

Feminino

Masculino

Prática de exercícios físicos

Sim

Não

\section{Função remunerada}

Sim

Não

\section{Religião}

Sim

Não

Bebidas alcoólicas por pais ou responsáveis

Sim

Não

\section{Relacionamento com a mãe}

Não tenho mãe

Bom

Regular

Ruim

Não tenho contato

\section{Relacionamento com o pai}

Não tenho pai

Bom

Regular

Ruim

Não tenho contato

Classificação da classe socioeconômica

A 
Fonte: Dados da pesquisa.

Em relação ao CRAFFT/CESARE para rastreamento de uso de SPAs, a maioria dos adolescentes 72 (72.0\%) apresentou risco negativo, todavia $11(11.0 \%)$ apresentam risco para o uso, abuso ou dependência de SPAs, 6 (6.0\%) suspeita de dependência de SPAs e 2 (2.0\%) risco de dependência de SPAs.

No que se refere à preferência por estágios do desenvolvimento moral no dilema do médico, houve predomínio do estágio 2 (36\%), seguido pelo estágio 1 (23\%) e menor preferência pelo estágio 5 (4\%). No dilema do juiz, os estágios 5 e 6 destacaram-se com $23 \%$ e $25 \%$, respectivamente. E no dilema do operário, o estágio 4 foi o mais predominante com $42 \%$, seguido pelo estágio 3, com 20\% (Figura 1). Nossos resultados também mostram que o Índice C médio dos dilemas do médico, juiz e operário correspondeu a 31.94, 35.87, 41.83 pontos, respectivamente. A média geral foi 38.55 pontos.

Figura 1 - Porcentagem da preferência por estágios do desenvolvimento moral referente ao dilema do Médico (A), Juiz (B) e Operário (C).

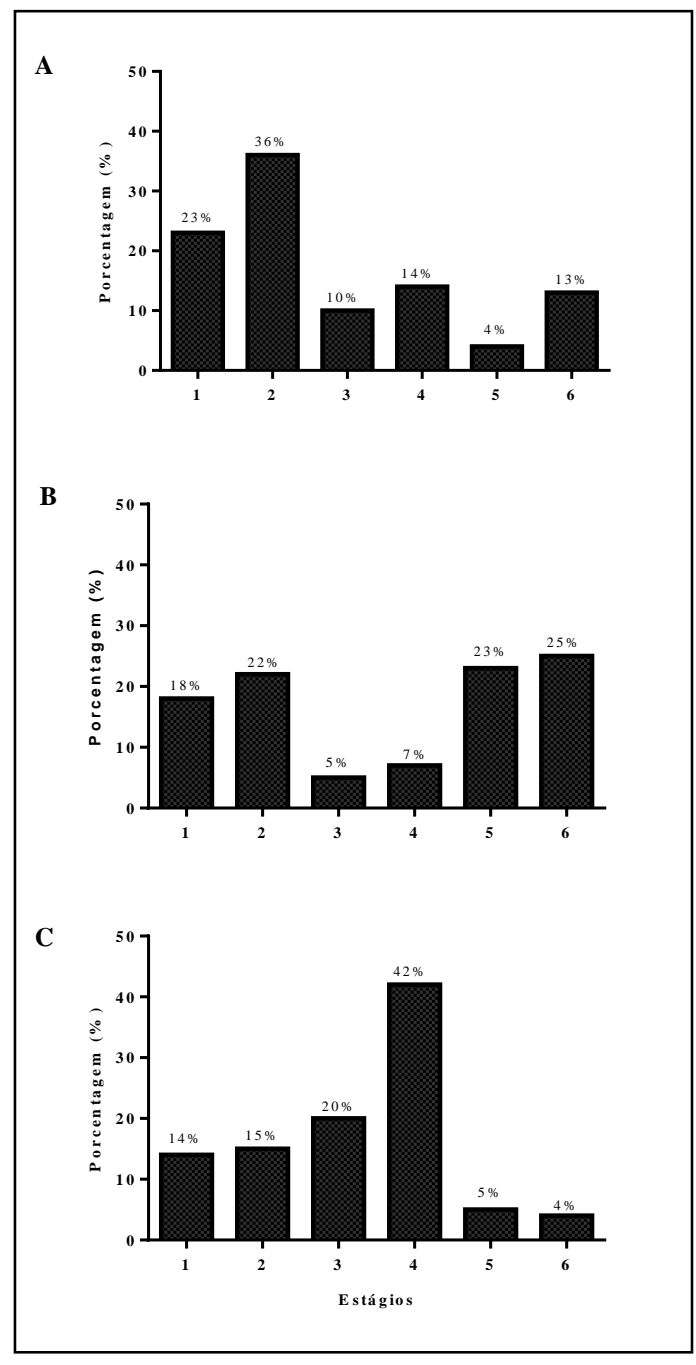

Fonte: Dados da pesquisa.

Quanto à clusterização dos dados, adotou-se cinco grupos, pois corresponde ao número de notas atribuídas pelo CRAFFT/CESARE. Dessa forma, a análise dos algoritmos foi realizada com base nos três cenários descritos na seção anterior, os quais variam o número de atributos. A Tabela 2 mostra os resultados das métricas apenas para o cenário 3 que apresenta total de atributos maior (87 atributos), por apresentar os melhores valores para as métricas selecionadas. Em relação aos 
resultados computacionais considerando as medidas (SSW, SSB e Silhouette) e os algoritmos (K-means, K-medoides e Fuzzy C-Means) pode-se verificar que o FCM atingiu o melhor desempenho para média do SSW (14859.057), SSB (1329.26722) e Silhouette (-0.01408749).

Tabela 2 - Comparativo dos três algoritmos em relação as medidas de avaliação para o cenário 3 com 87 atributos.

\begin{tabular}{lccc}
\hline & K-MEANS & K-MEDOIDS & FCM \\
\hline SSW médio & 1718015.86 & 2258369.77 & 14859.057 \\
SSB médio & 14091.9181 & 14859.057 & 1329.26722 \\
Silhouette médio & 0.29241514 & 14859.057 & -0.01408749 \\
\hline
\end{tabular}

Legenda: FCM (Fuzzy C-Means); SSW (sum of squares within clusters), SSB (sum of squares between clusters). Fonte: Dados da pesquisa.

O FCM atingiu sempre o mesmo resultado para todas as rodadas. Por outro lado, os outros algoritmos (K-means, Kmedoides) tiveram valores de métricas variados, por isso foram rodados 30 vezes. A dispersão destes pode ser vista na Figura 2, por meio do gráfico box plot, em que os números 1, 2 e 3 seguem a ordem descrita na seção anterior quanto a quantidade de atributos (81, 84 e 87), respeitando os cenários descritos. Era esperado que o K-medoides atingisse melhores desempenhos por ser influenciado menos com a questão da inicialização da posição dos centroides. Todavia, o K-means foi superior para os três cenários quando comparado com o K-medoides, por apresentar valores menores do SSW. Além disso, a Figura 2 deixa claro que, ao contrário do que se observou com o FCM, a inclusão de novos atributos deteriorou a qualidade da resposta, levando a SSWs maiores. Isto tem relação direta com a maior dificuldade imposta ao algoritmo para encontrar uma solução quando a tarefa se torna mais difícil.

Figura 2 - Box-Plot das funções K-means e K-medoides para os três cenários do estudo.

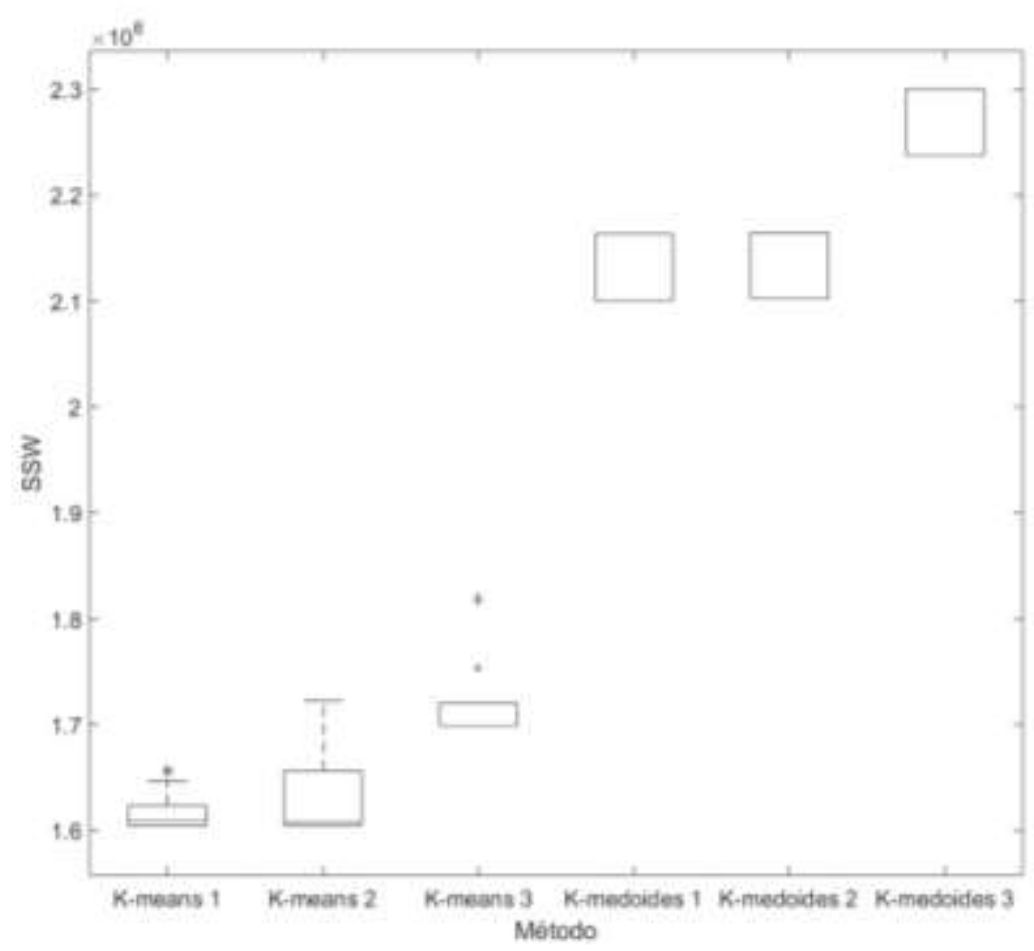

Fonte: Dados da pesquisa. 
Por fim, a Tabela 3 mostra a assertividade (percentual de acertos) dos algoritmos para cada grupo em relação às notas obtidas no CRAFFT/CESARE. O termo assertividade é relativo ao percentual dos valores dos algoritmos propostos que se enquadram exatamente no resultado do CRAFFT/CESARE. O algoritmo que apresentou melhor assertividade foi o FCM, com $100.00 \%$ no grupo 2 , seguido de $72.22 \%$ no grupo 1 e $66.60 \%$ no grupo 3 . Os grupos 4 e 5 , que representam suspeita de dependência de SPA's e risco de dependência de SPA's, apresentaram assertividade de $10.34 \%$ e $6.45 \%$, respectivamente.

Tabela 3 - Assertividade (percentual de acertos) dos algoritmos para cada grupo em relação ao CRAFFT/CESARE.

\begin{tabular}{lccccc}
\hline & Grupo 1 & Grupo 2 & Grupo 3 & Grupo 4 & Grupo 5 \\
\hline k-means & 70.31 & 25.00 & 0.00 & 11.76 & 9.09 \\
k-medoides & 20.00 & 13.30 & 5.26 & 100.00 & 5.00 \\
FCM & 72.22 & 100.00 & 66.60 & 10.34 & 6.45
\end{tabular}

Legenda: FCM (Fuzzy C-Means); SSW (sum of squares within clusters), SSB (sum of squares between clusters). Fonte: Dados da pesquisa.

\section{Discussão}

O início do uso de SPAs geralmente ocorre na adolescência intermediária (13 a 15 anos), enquanto o início do uso de álcool ocorre na fase inicial (de 10 a 12 anos). Isso explica parcialmente o aumento do uso de SPAs por estudantes na faixa etária de 16 a 19 anos (Andrade et al., 2017; Horta et al., 2018). A ingesta de álcool é uma prática, de certa forma, bem aceita e estimulada por grande parte da sociedade, e é compreensível a curiosidade do adolescente de aventurar-se em algo "tão adulto", mas, o consumo excessivo de álcool pode ser preditor ao uso de SPAs nesse grupo etário (Raposo et al., 2017).

Nossos resultados mostram que a maioria dos adolescentes entrevistados praticam atividade física e alguma religião, sendo estas práticas considerada como protetivas contra o uso de SPAs, podendo justificar o número baixo de estudantes que acusaram já ter usado SPAs. No que se refere a prática de exercícios físicos, o convívio proporcionado pelos esportes pode atuar de forma a diminuir/impedir o uso de SPAs, visto pelo propósito estético e saudável que tal prática estimula, e pelas amizades que se formam (Jorge et al., 2018; Nasser et al., 2016). Em relação a prática religiosa, por oferecer um sistema de condutas morais que visam, teoricamente, respeitar a vida, o corpo, o mundo e a sociedade, atribui-se baixo índice do uso de SPAs em pessoas ligadas a algum tipo de fé (Raposo et al., 2017; Benincasa, Resende, Heleno, \& Custódio, 2017; Santos et al., 2015).

No que concerne à família, ela pode ser considerada um dos pilares mais decisivos para um bom desenvolvimento moral do adolescente e para uma proteção quanto ao uso de SPAs. Por sua vez, o distanciamento familiar, episódios de violência doméstica e a utilização de SPAs pelos pais predispõe maior consumo de álcool e outras drogas por parte do adolescente (Andrade et al., 2017; Horta et al., 2018; Malta et al., 2018; Paiva et al., 2015). Dados sugerem que durante a adolescência, filhos de alcoólatras, apresentam um maior risco de uso nocivo de álcool e outras drogas, sendo de até quatro vezes maior do que os filhos de não alcoólatras, colocando, por vezes, o adolescente em situações de risco ou ilegais (Andrade et al., 2017; Chein, Albert, O’Brien, Uckert, \& Steinberg, 2011).

Em nosso estudo, mesmo com mais da metade dos pais e/ou responsáveis utilizando bebidas alcoólicas, a maioria dos adolescentes não apresentou fator de risco para o uso ou abuso de SPAs, mostrando que o bom relacionamento com a família, em especial os pais ou responsáveis, é importante para a proteção do adolescente contra o uso de SPAs, corroborando com nossos resultados que a maioria relatou ter bom relacionamento com pais e/ou mãe.

Quanto a classificação socioeconômica as classes mais inferiores prevaleceram, em virtude do estudo ter sido realizado em uma escola pública. A classe econômica do indivíduo pode ser um fator precursor, mas não determinante, do uso de SPAs (Shackleton, Milne, \& Jerrim, 2019). O uso de álcool e outras drogas na adolescência e a camada social não são 
consoantes quanto a associação entre menos privilégios e mais drogas. Na Europa, a variação dessa relação entre os países é imensa, e essa relação pode ser válida apenas quando o nível de pobreza é extremo e está ligado a problemas de comportamento, como o furto, por exemplo (Hormenu, Júnior, \& Schack, 2018; Martins-Oliveira, Ferreira, Vale, Ferreira, \& Zarzar, 2016).

No que se refere ao Índice C médio, todos os dilemas (médico, juiz e operário) apresentaram nível alto de competência moral (acima de 30 pontos). Esse padrão na pontuação do Índice C médio corrobora com a de outros países envolvendo também dilemas da Eutanásia e do Juiz (quantidade de vidas vs. respeito a uma vida). Uma pesquisa realizada na China encontrou Índice C médio de 31,4 pontos e na Alemanha, em torno de 40. Diferente nos EUA, que apresentou 23,8 pontos e no Irã, 20 (Liquat, 2012; Akin, 2018).

Além da análise descritiva do perfil dos estudantes apresentado acima, o estudo utilizou também análise envolvendo simulações computacionais, a clusterização, com o intuito de proporcionar maior precisão e acurácia nos resultados. A clusterização apresenta-se como um dos melhores métodos de análise de dados, além de ser útil em uma miríade de áreas do conhecimento, principalmente na saúde. No Brasil, tem sido utilizado nas áreas de biologia, psicologia, saúde coletiva, dentre outras, entretanto, no que se refere à relação entre adolescentes, desenvolvimento moral e uso de SPAs, o presente trabalho é inédito (Treichel, Jardim, Kantorski, Vasem, \& Neutzling 2016; Dharmarajan \& Velmurugan, 2015).

A literatura aponta escassez de publicações que utilizem os agrupamentos em cluster quando o assunto são adolescentes e o uso de álcool e outras drogas. Destaca-se um trabalho americano a respeito da prevenção do uso de cigarro e bebidas alcoólicas por adolescentes, que utilizou a clusterização pelos métodos de métrica de distância euclidiana ao quadrado e cluster de variância mínima de Ward (Babbin, Velicer, Paiva, Brick, \& Redding, 2015).

Nesse sentido, nossos resultados mostram que os melhores resultados foram atribuídos pelo FCM e que a discordância entre os grupos com o teste CRAFFT/CESARE mostra que não há preditores definitivos do uso de SPAs. Entretanto, observase um grau de similaridade nas repostas de ambos os métodos para os maiores grupos, da ordem de 72,22\% e 100\%, o que corrobora os a hipótese que tais ferramentas são complementares e devem ser consideradas em conjunto. Esta investigação abre novas perspectivas para a utilização de ferramentas computacionais robustas em problemas reais como o aqui abordado.

Nota-se que simplesmente aumentar a quantidade de dados de entrada (atributos) não trouxe benefícios quanto ao agrupamento, quando se considera a totalidade dos métodos utilizados. Isto pode ser explicado pois quanto mais atributos, maior o espaço de busca a ser investigado, o que pode estar diminuindo a capacidade de otimização dos mesmos. Entretanto, tal aumento foi benéfico para o FCM.

\section{Considerações Finais}

Nossos resultados da pesquisa revelaram que a maioria dos adolescentes possui entre 16 e 18 anos de idade, são do sexo feminino, exercem função remunerada, praticam atividade física e alguma religião. A maioria dos pais ou responsáveis dos adolescentes consumem bebidas alcoólicas e relatam ter bom relacionamento bom principalmente com a mãe. As classes econômicas mais inferiores prevalecem entre os entrevistados. Em relação a preferência pelos estágios do desenvolvimento moral, no dilema do médico, houve predomínio dos estágios 1 e 2, no dilema do juiz, os estágios 5 e 6 . E no dilema do operário, os estágios 4 e 5 . Todos os dilemas (médico, juiz e operário) apresentaram nível alto de competência moral (acima de 30 pontos). Ademais, a aplicação de ferramentas de clusterização, sobretudo o método Fuzzy C-Means mostrou-se adequada, com alta correlação com as respostas fornecidas pelo teste CRAFFT/CESARE. Isto abre novas perspectivas para análise de dados como a aqui abordada.

Considera-se, portanto, que este estudo possui grande contribuição relacionada ao uso de substâncias psicoativas e o desenvolvimento moral de adolescentes, além da neurociência, pois ajuda a compreender os fatores determinantes na tomada 
de decisão e/ou julgamento nessa etapa da vida, contemplando algumas lacunas apontadas na literatura. Considera-se, portanto, que os achados possam contribuir para melhor compreensão do perfil do adolescente com predisposição ao uso de SPAs, e assim desenvolver possibilidades e estratégias de intervenções para esse público.

Como sugestão para próximas produções científicas que abordarão essa temática, propõe-se que os estudantes de escolas privada também sejam incluídos na amostra, além da investigação quanto a etnia do entrevistado para que seja possível realizar outras correlações. Ademais, a forma de aplicação dos questionários também pode ser inovada, como o uso dos aplicativos de celulares e/ou computadores, os quais trazem mais sigilo quanto a identidade dos participantes.

\section{Referências}

Akin, A. (2018). Religious Education and Moral Development: The influence of a religious childhood on moral orientation and competence. Ethics in Progress, 9(2), 27-43.

Andrade, M. E. D., Santos, I. H. F., Souza, A. A. M. D., Silva, A. C. S., Leite, T. D. S., Oliveira, C. C. D. C., \& Albuquerque Júnior, R. L. C. D. (2017). Experimentação de substâncias psicoativas por estudantes de escolas públicas. Revista de saúde pública, $51,82$.

Babbin, S. F., Velicer, W. F., Paiva, A. L., Brick, L. A. D., \& Redding, C. A. (2015). Replicating cluster subtypes for the prevention of adolescent smoking and alcohol use. Addictive behaviors, 40, 57-65.

Brasil (2017). SUPERA: Sistema para detecção do Uso abusivo e dependência de substâncias Psicoativas: Encaminhamento, intervenção breve, Reinserção social e Acompanhamento. Brasília: Secretaria Nacional de Políticas sobre Drogas.

Bataglia, P. U. R. (2010). A validação do Teste de Juízo Moral (MJT) para diferentes culturas: o caso brasileiro. Psicologia: reflexão e crítica, $23(1)$, 83-91.

Bataglia, P. U. R., Morais, A. D., \& Lepre, R. M. (2010). A teoria de Kohlberg sobre o desenvolvimento do raciocínio moral e os instrumentos de avaliação de juízo e competência moral em uso no Brasil. Estudos de Psicologia (Natal), 15(1), 25-32.

Benincasa, M., Resende, M. M., Heleno, M. G. V., \& Custódio, E. M. (2017). Qualidade de vida e resiliência em estudantes do ensino médio: um estudo epidemiológico na cidade de São Paulo. Psicologia Revista, 26(2), 363-383.

Campos, J. A. D. B., Almeida, J.C., Garcia, P. P. N. S., \& Faria, J.B. (2011). Consumo de álcool entre estudantes do ensino médio do município de Passos MG. Revista Ciência e Saúde Coletiva, 16 (12), 4745-4754.

Carbonário, F.A. (2018). Neurociência do abuso de drogas na adolescência. Revista Mental, 12 (22), 114-117.

Chein, J., Albert, D., O’Brien, L., Uckert, K., \& Steinberg, L. (2011). Peers increase adolescent risk taking by enhancing activity in the brain's reward circuitry. Developmental Science, 14 (2), 1-10.

Cohen, A.O., \& Casey, B.J. (2014). Rewiring juvenile justice: the intersection of developmental neuroscience and legal policy. Trends Cognitive Sciences, 18 (2), 63-65.

Colver, A., \& Longwell, S. (2013). New understanding of adolescent brain development; relevance to transitional healthcare for young people with long term conditions. Arch Dis Child, 98 (11), 902-907.

Coutinho, E. S. F., França- Santos, D., Magliano, E. S., Bloch, K. V., Barufaldi, L. A., Cunha, C. F., Vasconcellos, M. T. L., \& Szklo, M. (2016). ERICA: Padrões de consumo de bebidas alcoólicas em adolescentes brasileiros. Revista Saúde Pública, 50 (1).

Dharmarajan, A., \& Velmurugan, T. (2015). Lung cancer data analysis by k-means and farthest first clustering algorithms. Indian Journal Science Technology, $8(15), 1-8$.

Figueiredo, E., Macedo, M., Siqueira, H. V., Santana, J. R. C. J., Gokhale, A., \& Bastos-Filho, C. J. A. (2019). Swarm intelligence for clustering: A systematic review with new perspectives on data mining. Engineering Applications of Artificial Intelligence, (82), 313-329.

Freitas, L. M. F. D., \& Souza, D. P. O. D. (2020). Prevalência do uso de drogas e relações familiares entre adolescentes escolares de Cuiabá, Mato Grosso: estudo transversal, 2015. Epidemiologia e Serviços de Saúde, 29, e2019118.

Garanito, M. P., \& Zaher-rutherford, V. L. (2019). O paciente adolescente e a deliberação clínica sobre a sua saúde. Revista Paulista de Pediatria, 37 (4), 503509.

Horta, R. L., Mola, C. L., Horta, B. L., Mattos, C. N. B., Andreazzi, M. A. R., Oliveira- Campos, M., \& Malta, D. C. (2018). Prevalence and factors associated with illicit drug use throughout life: National School Health Survey 2015. Revista Brasileira de Epidemiologia, 1 (21).

Hormenu, T., Júnior, J. E. H., \& Schack, T. (2018). Predictors of alcohol consumption among inschool adolescents in the Central Region of Ghana: A baseline information for developing cognitive-behavioural interventions. PLoS One,13 (11).

Jorge, K. O., Ferreira, R. C., Ferreira, E. F., Kawachi, I., Zarzar, P. M., \& Pordeus, I. A. (2018). Peer group influence and illicit drug use among adolescent students in Brazil: a cross-sectional study. Caderno de Saúde Pública, 3 (34).

Kohlberg, L. (1981). Essays on Moral Development: The psychology of moral development. São Francisco: Harper e Row. 
Lind, G. (2000). O significado e medida da competência moral revisitada: um modelo do duplo aspecto da competência moral. Revista Psicologia: Reflexão e Crítica, 13 (3), 399-416.

Lind, G. (2015). Scoring and interpreting the Moral Competence Test (MCT) - an introduction. Konstanz: University of Konstanz. http://zip.net/bpsL1j.

Liquat, A. W. (2012). Effect of dogmatic religiosity and educational environment on moral judgment competence [tese]. Department of Psychology, Faculty of Social Sciences, International Islamic University Islamabad.

Malta, D. C., Machado, I. E., Felisbino-Mendes, M. S., Prado, R. R., Pinto, M. A. S., Oliveira-Campos, M., Souza, M. F. M., \& Assunção, A. A. (2018). Use of psychoactive substances among Brazilian adolescents and associated factors: National School-based Health Survey, 2015. Revista Brasileira de Epidemiologia, (1).

Marriel, L. C., Assis, S. G., Avanci, J. Q., \& Oliveira, R. V. C. (2013). Violência escolar e autoestima de adolescentes. Caderno Pesquisa, 36 (127), $35-50$.

Martins-Oliveira, J.G., Ferreira, R.C., Vale, M.P., Ferreira, E. F., \& Zarzar, P. (2016). Risco De Dependência Do Álcool: Prevalência, Problemas Relacionados E Fatores Socioeconômicos. Revista Ciência e Saúde Coletiva, 21 (1), 17-26.

Nunes, V. B., \& Bataglia, P. U. R. (2019). Trabalho com dilemas morais na resolução de conflitos entre escolares: fundamentos habermasianos à teoria kohlberguiana. Revista de Educação PUC-Campinas 2019, 24 (1), 125-138.

Nasser, R. L., Branco, J. C., Lara, D. R., Del Vecchio, F. B., Wienner, C., Mesquita, P., Souza, L.D.M., Silva, R. A., \& Jansen, K. (2016). Atividade física de lazer e uso de substâncias lícitas em uma amostra populacional de adultos jovens. Ciência \& Saúde Coletiva, 21 (1).

Paiva, P. C. P., Paiva, H. N., Lamounier, J. A., Ferreira, E. F., César, C. A. S., \& Zarzar, P. M. (2015). Consumo de álcool em binge por adolescentes escolares de 12 anos de idade e sua associação com sexo, condição socioeconômica e consumo de álcool por melhores amigos e familiares. Revista Ciência e Saúde Coletiva, 20 (11), 3427-3435.

Pagliace, A. G. S., Maftum, M. A., Lacerda, M. R., Kantorski, L. P., Nimtz M. A., \& Brusamarello T. (2019). Avaliação do cuidado às crianças e aos adolescentes usuários de substâncias psicoativas: potencialidades e fragilidades. Texto \& Contexto Enfermagem 2019, 18 (3).

Pereira, A. S. et al. (2018). Metodologia da pesquisa científica. UFSM. https://repositorio.ufsm.br/bitstream/handle/1/15824/Lic_Computacao_MetodologiaPesquisa-Cientifica.pdf?sequence $=1$

Pereira, B. A. A. X., \& Azevedo, R. C. S. (2019). Desafio na vida real: capacitação sobre uso de drogas e adolescência na atenção básica. Revista Saúde Pública, 53 (4).

Pereira, B. A. A. X., Schram, P. F. C., \& Azevedo, R. C. S. (2016). Avaliação da versão brasileira da escala CRAFFT/ CESARE para uso de drogas por adolescentes. Revista Ciência e Saúde Coletiva, 21 (1), 91-99.

Ramos, J. C. S. D. (2019). O cérebro adolescente em formação [tese]. Rio de Janeiro: Universidade Candido Mendes.

Raposo, J. C. S., Costa, A.C.Q., Valença, P. A. M., Zarzar, P. M., Diniz, A. S., Colares, V., \& Franca, C. (2017). Uso de drogas ilícitas e binge drinking entre estudantes adolescentes. Revista de Saúde Pública, 51 (83).

Santos, A.R.M., Oliveira, L. M. F. T., Júnior, J. C. F., Silva, P. P. C., Silva, E. A. P. C., \& Freitas, C. M. S. M. (2015). Associação entre prática religiosa e comportamentos de risco à saúde em adolescentes de Pernambuco. Revista Brasileira de Atividade Física e Saúde, 20 (3), 284-296.

Schlindwein-zanini, R., \& Sotili, M. (2019). Uso de drogas, repercussões e intervenções neuropsicológicas. Caderno Brasileiro de Saúde Mental, 11 (28), 94 116.

Schoen-Ferreira, T. H, Aznar-farias, M., \& Silvares, E. F. (2010). Adolescência através dos séculos. Psicologia: Teoria.e Pesquisa, 26 (2),227-234.

Shackleton, N., Milne, B.J., \& Jerrim, J. (2019). Socioeconomic Inequalities in Adolescent Substance Use: Evidence from Twenty-Four European Countries. Substance Use and Misuse, 54(6), 1044-1049.

Treichel, C. A.D.S., Jardim, V.M.D.R., Kantorski L. P., Vasem, M. L., \& Neutzling, A. D. S. (2016). Clustering of minor psychiatric disorders and burden among family caregivers of individuals with mental illness. Revista Ciência e Saúde Coletiva, 21 (2), 585-590.

Velho, M. T.A. C., Quintana, A. M., \& Rossi, A. G. (2014). Adolescência, autonomia e pesquisa em seres humanos. Revista Bioética, 22 (1), 76-84. 